Article

\title{
Rickettsia Detected in the Reptile Tick Bothriocroton hydrosauri from the Lizard Tiliqua rugosa in South Australia
}

\author{
Harriet Whiley ${ }^{1,+}$, Georgie Custance ${ }^{2,+}$, Stephen Graves ${ }^{3}$, John Stenos ${ }^{3}$, Michael Taylor ${ }^{1}$, \\ Kirstin Ross ${ }^{1}$ and Michael G. Gardner ${ }^{2,4, *}$ \\ 1 School of the Environment, Health and the Environment, Flinders University, GPO BOX 2100, Adelaide 5001, \\ Australia; harriet.whiley@flinders.edu.au (H.W.); michael.taylor@flinders.edu.au (M.T.); \\ kirstin.ross@Flinders.edu.au (K.R.) \\ 2 School of Biological Sciences, Flinders University, GPO Box 2100, Adelaide 5001, Australia; \\ cust0014@flinders.edu.au \\ 3 Australian Rickettsial Reference Laboratory, Barwon Health, Geelong Hospital, PO BOX 281, Geelong 3220, \\ Australia; graves.rickettsia@gmail.com (S.G.); JOHNS@barwonhealth.org.au (J.S.) \\ 4 Evolutionary Biology Unit, South Australian Museum, North Terrace, Adelaide 5000, Australia \\ * Correspondence: michael.gardner@flinders.edu.au; Tel.: +61-8-201-2315; Fax: +61-8-201-3015 \\ + These authors contributed equally to this work.
}

Academic Editor: Lawrence S. Young

Received: 27 January 2016; Accepted: 7 June 2016; Published: 8 June 2016

\begin{abstract}
Rickettsiosis is a potentially fatal tick borne disease. It is caused by the obligate intracellular bacteria Rickettsia, which is transferred to humans through salivary excretions of ticks during the biting process. Globally, the incidence of tick-borne diseases is increasing; as such, there is a need for a greater understanding of tick-host interactions to create more informed risk management strategies. Flinders Island spotted fever rickettsioses has been identified throughout Australia (Tasmania, South Australia, Queensland and Torres Strait Islands) with possible identifications in Thailand, Sri Lanka and Italy. Flinders Island spotted fever is thought to be spread through tick bites and the reptile tick Bothriocroton hydrosauri has been implicated as a vector in this transmission. This study used qPCR to assay Bothriocroton hydrosauri ticks collected from Tiliqua rugosa (sleepy lizard) hosts on mainland South Australia near where spotted fever cases have been identified. We report that, although we discovered Rickettsia in all tick samples, it was not Rickettsia honei. This study is the first to use PCR to positively identify Rickettsia from South Australian Bothriocroton hydrosauri ticks collected from Tiliqua rugosa (sleepy lizard) hosts. These findings suggest that $B$. hydrosauri may be a vector of multiple Rickettsia spp. Also as all 41 tested B. hydrosauri ticks were positive for Rickettsia this indicates an extremely high prevalence within the studied area in South Australia.
\end{abstract}

Keywords: Rickettsia; Flinders Island spotted fever; rickettsioses; Bothriocroton hydrosauri; Tiliqua rugosa; tick; PCR

\section{Introduction}

Worldwide, the incidence of tick borne disease is increasing [1]. This includes tick borne rickettsiosis which is caused by the obligate intracellular bacteria belonging to the Rickettsia genus [2]. Rickettsiosis in humans is a potentially fatal febrile illness that can be categorised as scrub typhus, typhus group and spotted fever group rickettsioses [3]. Presently, 16 of the 25 known Rickettsia species have been identified as human pathogens [4]. Rickettsia are present in the tick salivary excretions and transferred to humans during the tick biting process [3]. In Australia, Flinders Island spotted fever is an Australian tick borne disease caused by R. honei (also known as strain TT-118). It was first discovered 
on Flinders Island, Tasmania, but has since been detected in clinical patients from other parts of Australia, including in South Australia [5,6]. There has also been a reported cases of Flinders Island spotted fever in Nepal [7]. Rickettsia honei has also been detected in Thailand from larval Ixodes and Rhipicephalus ticks and I. granulatus from Rattus rattus and in Texas, USA, from Amblyomma cajennense ticks taken from cattle. However, the human pathogenicity of $R$. honei in Thailand and USA has not been confirmed [8].

Previously, Stenos et al. [9] examined reptile ticks Bothriocroton hydrosauri from Flinders Island, Australia, and detected Rickettsia via PCR in 63\% (29/46) ticks. Subsequent sequencing of seven cell culture samples confirmed a 100\% match to $R$. honei. The ticks had been collected from reptile hosts including 12 Tiliqua nigrolutea (blue tongue lizards), four Agkistrodon contortic (copperhead snakes) and three Notechis scutatus (tiger snakes). All blood samples collected from reptile hosts were negative for Rickettsia.

In South Australia, there have been confirmed cases of $R$. honei isolated from patients in the same geographical location known for B. hydrosauri [10]. However, $R$. honei has not been previously identified from South Australian B. hydrosauri but is considered likely to occur in this tick species due to the proximity of the disease and the ticks. This study used PCR to test for the presence of Rickettsia in B. hydrosauri collected from Tiliqua rugosa (the sleepy lizard) hosts found in the South Australian Murray Mallee (SAMM) region.

\section{Results and Discussion}

All of the $41 B$. hydrosauri ticks tested were positive for Rickettsia DNA. We suspected the species would be R. honei, however, all of the eight sequenced ompA products (KX290293-KX290300) had 99\% homology with an undescribed species (accession number: EU283837). The closest match with a named species was 95\% with Rickettsia tamurae (accession number: DQ103259.1). Using PCR, the DNA from the 15 T. rugosa hosts blood samples were all negative.

This study is the first to positively identify Rickettsia from South Australian B. hydrosauri ticks but instead of the expected agent of Flinders Island Fever, $R$. honei we uncovered potentially a different, as yet uncharacterized, species. Our study has several important findings. First, it indicates that the reptile tick B. hydrosauri is possibly not the host of $R$. honei in the sampled area. Second, our results implicates $B$. hydrosauri as a potential vector of other species of Rickettsia and not just $R$. honei. Previous studies have only identified Rickettsia from B. hydrosauri ticks collected from Flinders Island, Tasmania [9].

Third, our findings indicate an extremely high prevalence of Rickettsia within the studied area in South Australia as all ticks tested were positive. Reports from Stenos [9] indicate a lower prevalence on Flinders Island, albeit of a different Rickettsia.

A forth outcome is our lack of detecting horizontal transmission of Rickettsia from tick to lizard and our results support vertical transmission although they do not exclude horizontal transmission being present. We did find that all life stages: adult, larval, and nymph; tested positive for Rickettsia. Transovarial transmission has been demonstrated for some Rickettsia species [11]. The presence of Rickettsia in the B. hydrosauri larvae further supports this mode of transmission. This supports the findings by Stenos et al. [9] that did not detect $R$. honei from any reptile hosts carrying Rickettsia positive ticks. The negative results could be due to the numbers of Rickettsia present in the blood sample being less than the limit of detection or that T. rugosa is not a main host. Further work on tissues would be required to determine if Rickettsia remain viable, but quiescent, in any organ of the lizards.

Although further work would be required to identify if this species of Rickettsia causes disease, as B. hydrosauri are known to feed on humans [12], our results indicate a greater potential for humans to be exposed to a potential vector of disease than previously thought. This reptile and tick association occurs across a large area of Southern Australia, particularly in South Australia in rural areas close to Adelaide. Further work would be required to understand if the prevalence in the studied area is mirrored elsewhere in the ticks range in South Australia or in other tick species or indeed if the same 
species of Rickettsia is involved in different parts of the tick and lizards range. Future studies comparing these samples to clinical isolates would provide insight into the epidemiological significance of these results and how the prevalence of Rickettsia in B. hydrosauri hosts affects the incidence of spotted fever rickettsiosis in humans.

Finally, we have potentially uncovered an unnamed species of Rickettsia which has previously only been reported from the reptile tick Amblyomma fimbriatum [13] from the Northern Territory of Australia, thus extending the range and potential vectors of this Rickettsia. Confirmation of this species would require isolation and further genomic characterisation. The disease causing nature of this species also needs to be confirmed.

\section{Experimental Section}

\subsection{Sample Collection}

We utilized 35 adult male, 1 adult female, 3 nymphs and 2 larval B. hydrosauri ticks harvested from 35 T. rugosa hosts captured from the South Australian Murray Mallee (SAMM) region during a previous study [14]. Sampling was conducted in the hot and dry summer months of November to February on days when the ground temperature was $<28^{\circ} \mathrm{C}$. We also sampled blood from the caudal vein of a subset of 15 lizard hosts.

\subsection{DNA Extraction}

DNA was extracted from 41 whole ticks using the PowerSoil ${ }^{\circledR}$ DNA Isolation Kit MO BIO Laboratories, Inc., Carlsbad, CA, USA) following manufactures instructions, additionally incorporating homogenization by $0.1 \mathrm{~mm}$ zirconium bead-milling to disrupt bacteria. DNA extraction from the blood of lizards followed [14].

\subsection{PCR Detection}

Rickettsia specific qPCR was performed using the Rotor-Gene ${ }^{\circledR}$ SYBR ${ }^{\circledR}$ Green PCR Kit (Qiagen, Victoria, Australia) and previously described primers [15]. All samples were tested in duplicate. The $25 \mu \mathrm{L}$ reactions contained $12.5 \mu \mathrm{L}$ of Rotor-Gene SYBR Green PCR Master Mix, $5 \mu \mathrm{L}$ RNase-Free Water, $1 \mu \mathrm{M}$ CS-F (5'-TCG CAA ATG TTC ACG GTA CTT T-3'), $1 \mu \mathrm{M}$ CS-R (5'-TCG TGC ATT TCT TTC CAT TGT G-3') and $2.5 \mu \mathrm{L}$ of template DNA (ca. $20 \mathrm{ng}$ ). The cycling conditions included an initial hold at $95^{\circ} \mathrm{C}$ for $5 \mathrm{~min}$, followed by 40 cycles consisting of $95^{\circ} \mathrm{C}$ for $5 \mathrm{~s}$ and $60^{\circ} \mathrm{C}$ for $10 \mathrm{~s}$. All PCR reactions were carried in a RotorGene 3000 (Corbett Research, Sydney, Australia) with data acquisition at $60^{\circ} \mathrm{C}$ on the Green channel (excitation at $470 \mathrm{~nm}$, detection at $510 \mathrm{~nm}$ ) at a gain of 9.67. Melt curve data was also acquired on this channel using a ramping rate of $1^{\circ} \mathrm{C} / 90 \mathrm{~s}$ from $60^{\circ} \mathrm{C}$ to $95^{\circ} \mathrm{C}$. Each PCR run included a positive R. honei control (Australian Rickettsial Information Laboratory in Geelong, Victoria, Australia) and a non-template control of nuclease free water (Qiagen, Australia). For each reaction the melt curve was analysed and a positive Rickettsia result was confirmed with a melting temperature $\left(\mathrm{T}_{m}\right)$ of $78-79 \pm 1{ }^{\circ} \mathrm{C}$ as shown in Figure 1.

\subsection{Sequencing of Positive Samples}

We sequenced the PCR products from a subset of 10 positive tick samples to identify the Rickettsia species amplified. PCR products were purified and sequenced by the Australian Genome Research Facility Ltd (Adelaide, Australia) using previously described primers CS-F (5'-TCG CAA ATG TTC ACG GTA CTT T-3') and CS-R (5'-TCG TGC ATT TCT TTC CAT TGT G-3') [15]. After a positive match with Rickettsia with this small fragment, the samples were amplified with primers Rr190.70p (ATG GCG AAT ATT TCT CCA AAA) and Rr190.602n (AGT GCA GCA TTC GCT CCC CCT) [16] and eight PCR products were successfully sequenced. All sequences were analysed using the Basic Local Alignment Search Tool (BLAST) [17]. 


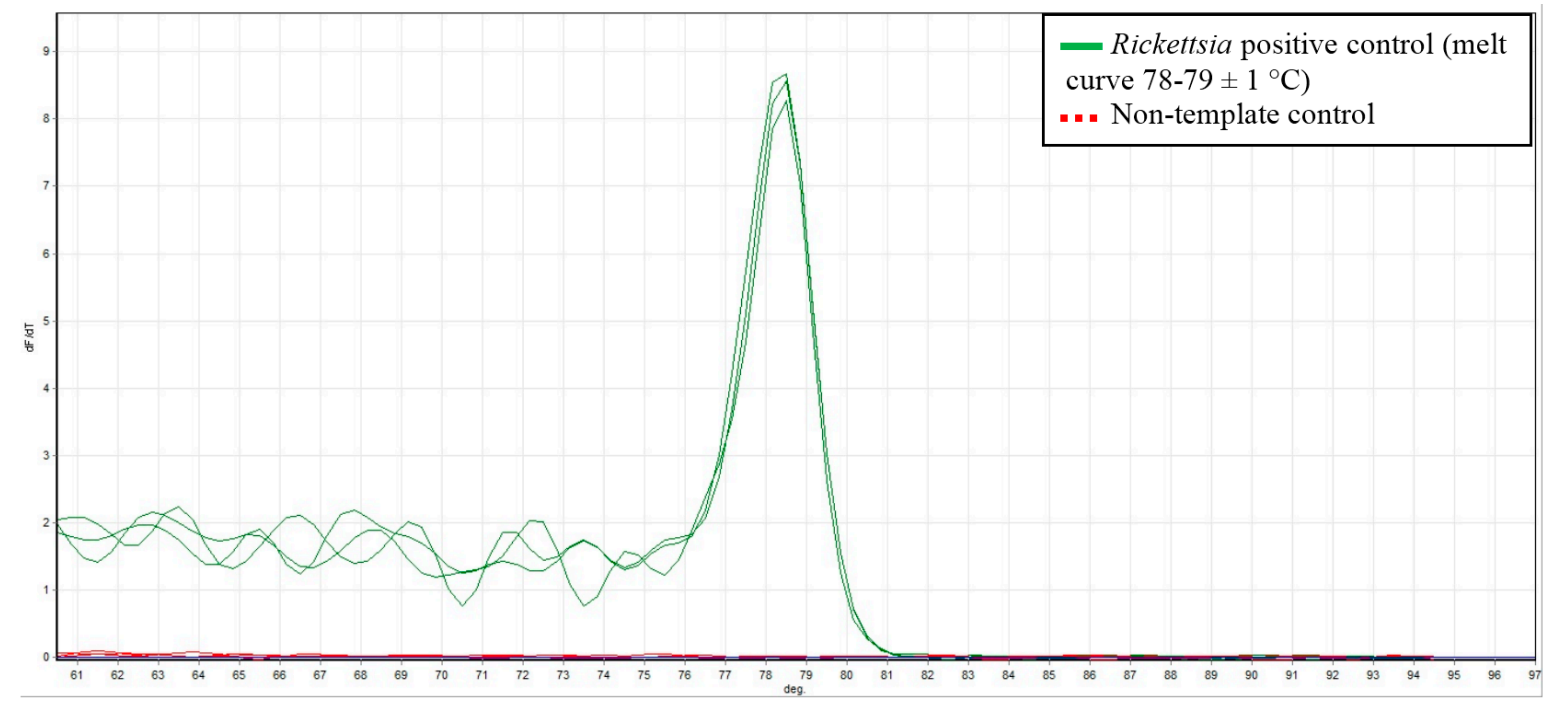

Figure 1. Melt curve data showing the Rickettsia positive control with a $\mathrm{T}_{m}$ of $78-79 \pm 1{ }^{\circ} \mathrm{C}$ (solid green line) and the no- template control (dashed red line).

\section{Conclusions}

This study provides an insight into the prevalence and variety of Rickettsioses in South Australia. Intriguingly, we did not uncover the causative agent of Flinders Island spotted fever, $R$. honei, but possibly a yet unnamed species. This study was the first to identify Rickettsia from South Australian B. hydrosauri ticks collected from T. rugosa (sleepy lizard) hosts. These findings identify B. hydrosauri as a potential vector of multiple Rickettsia. All T. rugosa hosts were negative for Rickettsia spp. which could be indicative of the limit of detection or could suggest the absence of horizontal transmission from tick to lizard host but does not exclude this form of transmission. This potential absence of horizontal transmission could be explored further through the testing of lizards using serology methods. Further work is also required to characterize the unnamed Rickettsia species found.

Acknowledgments: We thank Sheethal Prasad for initial laboratory work, Leanne McGrath at the AGRF for extracting the tick DNA, and Tara Garrard for troubleshooting laboratory problems. Anita Smyth, Liz Smee and Mina Ansari are thanked for collecting the ticks and lizard blood. Hazizul Hussain-Yusef for supplying the positive control. All work was conducted under appropriate permits.

Author Contributions: Harriet Whiley wrote the manuscript and conducted part of the laboratory work; Georgie Custance performed laboratory work; Kirstin Ross, Michael Taylor, John Stenos and Stephen Graves contributed intellectually to the work; Michael Gardner conceived the study and wrote the manuscript. All authors commented on manuscript drafts.

Conflicts of Interest: The authors declare no conflict of interest.

\section{References}

1. Dantas-Torres, F.; Chomel, B.B.; Otranto, D. Ticks and tick-borne diseases: A one health perspective. Trends Parasitol. 2012, 28, 437-446. [CrossRef] [PubMed]

2. Parola, P.; Paddock, C.D.; Socolovschi, C.; Labruna, M.B.; Mediannikov, O.; Kernif, T.; Abdad, M.Y.; Stenos, J.; Bitam, I.; Fournier, P.-E.; et al. Update on tick-borne rickettsioses around the world: A geographic approach. Clin. Microbiol. Rev. 2013, 26, 657-702. [CrossRef] [PubMed]

3. Derne, B.; Weinstein, P.; Musso, D.; Lau, C. Distribution of rickettsioses in oceania: Past patterns and implications for the future. Acta Trop. 2015, 143, 121-133. [CrossRef] [PubMed]

4. Fournier, P.E.; Raoult, D. Current knowledge on phylogeny and taxonomy of Rickettsia spp. Ann. N. Y. Acad. Sci. 2009, 1166, 1-11. [CrossRef] [PubMed]

5. Unsworth, N.B.; Stenos, J.; McGregor, A.R.; Dyer, J.R.; Graves, S.R. Not only 'Flinders Island'spotted fever. Pathology 2005, 37, 242-245. [CrossRef] [PubMed] 
6. Abdad, M.Y.; Cook, A.; Dyer, J.; Stenos, J.; Fenwick, S.G. Seroepidemiological study of outdoor recreationists' exposure to spotted fever group Rickettsia in Western Australia. Am. J. Trop. Med. Hyg. 2014, 91, 584-588. [CrossRef] [PubMed]

7. Murphy, H.; Renvoisé, A.; Pandey, P.; Parola, P.; Raoult, D. Rickettsia honei infection in human, Nepal, 2009. Emerg. Infect. Dis. 2011, 17, 1865. [CrossRef] [PubMed]

8. Graves, S.; Stenos, J. Rickettsia honei. Ann. N. Y. Acad. Sci. 2003, 990, 62-66. [CrossRef] [PubMed]

9. Stenos, J.; Graves, S.; Popov, V.L.; Walker, D.H. Aponomma hydrosauri, the reptile associated tick reservior of Rickettsia honei on Flinders Island, Australia. Am. J. Trop. Med. Hyg. 2003, 69, 314-317. [PubMed]

10. Dyer, J.R.; Einsiedel, L.; Ferguson, P.E.; Lee, A.S.; Unsworth, N.B.; Graves, S.R.; Gordon, D.L. A new focus of Rickettsia honei spotted fever in South Australia. Med. J. Aust. 2005, 182, 231-234. [PubMed]

11. Parola, P.; Davoust, B.; Raoult, D. Tick-and flea-borne rickettsial emerging zoonoses. Vet. Res. 2005, 36, 469-492. [CrossRef] [PubMed]

12. Barker, S.C.; Walker, A.R. Ticks of Australia. The species that infest domestic animals and humans. Zootaxa 2014, 3816, 1-144. [CrossRef] [PubMed]

13. Vilcins, I.-M.E.; Fournier, P.-E.; Old, J.M.; Deane, E. Evidence for the presence of Francisella and spotted fever group Rickettsia DNA in the tick Amblyomma fimbriatum (acari: Ixodidae), Northern Territory, Australia. J. Med. Entomol. 2009, 46, 926-933. [CrossRef] [PubMed]

14. Lancaster, M.L.; Gardner, M.G.; Fitch, A.J.; Ansari, T.H.; Smyth, A.K. A direct benefit of native saltbush revegetation for an endemic lizard (Tiliqua rugosa) in southern Australia. Aust. J. Zool. 2012, 60, 192-198. [CrossRef]

15. Regnery, R.L.; Spruill, C.L.; Plikaytis, B.D. Genotypic identification of rickettsiae and estimation of intraspecies sequence divergence for portions of two rickettsial genes. J. Bacteriol. 1991, 173, 1576-1589. [PubMed]

16. Rogall, T.; Wolters, J.; Flohr, T.; Bottger, E.C. Towards a phylogeny and definition of species at the molecular level within the genus Mycobacterium. Int. J. Syst. Evol. Microbiol. 1990, 40, 323-330. [CrossRef] [PubMed]

17. Altschul, S.F.; Gish, W.; Miller, W.; Myers, E.W.; Lipman, D.J. Basic local alignment search tool. J. Mol. Biol. 1990, 215, 403-410. [CrossRef] 